\title{
POUR UNE HISTOIRE DES IDÉES LINGUISTIQUES
}

L'histoire des théories linguistiques s'est considérablement développée depuis une quinzaine d'années. Deux revues y sont entièrement consacrées : Historiographia Linguistica (1974, Amsterdam, John Benjamins), Histoire Épistémologie Langage (1979, Presses universitaires de Vincennes). Des colloques internationaux ont lieu régulièrement et plusieurs sociétés se sont crées qui ont cette discipline pour activité. En France, après la création de la Société d'histoire et d'épistémologie des sciences du langage (1978), une unité associée au CNRS a été fondée au Département de recherches linguistiques de l'Université Paris 7 (UA 381, 1984), qui regroupe une trentaine de chercheurs. Cette équipe achève actuellement une Histoire des idées linguistiques, d'un millier de pages, à paraitre aux éditions Mardaga (Liège), fin 1989. Cette intense activité internationale a entraîné de nombreuses discussions méthodologiques et un profond remaniement des connaissances. Je vais tâcher d'exposer rapidement le point de vue sur le développement des savoirs et des idées linguistiques que nous avons construit en rédigeant cette histoire, dans le but d'intégrer ces nouvelles connaissances en une vision globale.

Le savoir linguistique est multiple et il débute naturellement dans la conscience de l'homme parlant. Il est épilinguistique ${ }^{1}$, non posé pour soi dans la représentation avant d'être métalinguistique, c'est-à-dire représenté, construit et manipulé en tant que tel. La continuité entre l'épilinguistique et le métalinguistique peut être comparée avec la continuité entre la perception et la représentation physique dans les sciences de la nature. Alors que ces dernières ont rompu très tôt avec la perception, dès la physique galiléenne, pour s'en éloigner sans cesse davantage, le savoir linguistique, lui, n'a rompu que sporadiquement

1. La notion est due à Antoine Culioli, cf. «A propos du genre en anglais contemporain ", Les langues modernes, 3, 1968, p. 40.

Revue de synthèse: IV S. Nos 3-4, juil.-déc. 1988. 
avec la conscience épilinguistique, lorsque les grammairiens postulent des éléments non manifestes pour expliquer les phénomènes observables ou dans le domaine du comparatisme, au xix $x^{e}$ siècle, avec les lois phonétiques et les reconstructions. Dans le domaine proprement grammatical, encore aujourd'hui ${ }^{2}$, il n'y a pas toujours de véritable solution de continuité, peut-être parce que le langage est un système régulé par sa propre image ${ }^{3}$. L'utilisation pédagogique de la grammaire la relie toujours à la conscience du locuteur. En tout état de cause, le cœur de notre propos est le savoir métalinguistique constitué et/ou en voie de constitution. Il peut être de quatre types. D'abord de nature spéculative, situé purement dans l'élément de la représentation abstraite. Ensuite de nature pratique, c'est-à-dire finalisé par la nécessité d'acquérir une maîtrise. Dans ce cas, il semble assez généralement déterminé par trois types de maîtrises : (a) la maîtrise de l'énonciation, par-là nous entendons la capacité d'un locuteur de rendre sa parole adéquate à un but donné, convaincre, représenter le réel, etc.; (b) la maîtrise des langues, parler et/ou comprendre une langue, qu'il s'agisse de la langue maternelle ou d'autres langues; (c) la maîtrise de l'écriture. Les maitrises donnent lieu à la constitution de techniques, c'est-à-dire de pratiques codifiées permettant d'obtenir à tout coup (existence de règles) ou le plus souvent, un résultat voulu; elles donnent également lieu à la formation de compétences spécifiques, susceptibles de recevoir un statut professionnel dans une société donnée (truchements/interprètes, scribes, poètes, rhéteurs, etc.). Les données factuelles permettent d'avancer la thèse de l'indépendance de ces types de savoir. L'histoire des savoirs linguistiques est justement faite de leurs développements, de leurs interactions, voire du passage de la thématisation de certains phénomènes d'un type à un autre. Comme le montre le développement de la conception des parties du discours dans la Grèce, mais aussi celui des théories médiévales ou de la grammaire générale, la spécificité de l'Occident a résidé, très tôt, dans la perméabilité entre deux types de savoirs, la logique et la grammaire, construits l'un sur la maîtrise de l'énonciation, l'autre sur celle des langues. Elle tient aussi à l'effort, toujours présent dans le long terme, quoique pas toujours dominant, de déplacer tous les savoirs linguistiques vers un type de savoir spéculatif.

2. De nombreux auteurs identifient la théorie linguistique à la représentation de la connaissance du locuteur; cf. Esa ITKONEN, Grammatical Theory and Metascience, Amsterdam, Benjamins, 1978; voir également Hans Martin GAUGER, Sprachbewusstsein und Sprachwissenschaft, Munich, R. Pipper, 1976. Il faut noter que la thèse a été soutenue pour les sciences sociales en général : cf. Peter WiNCH, The Idea of a Social Science, Londres, Routledge and Kegan Paul, 1958.

3. Cf. Gilles-Gaston Granger, Langages et épistémologie, Paris, Klincksieck, 1979, p. $118-137$. 
C'est cet effort qui provoque sporadiquement les discussions sur la "scientificité" de tel ou tel type de savoir linguistique. En matière de langage, la forme spéculative, cependant, n'a jamais été suffisamment dominante pour qu'on puisse rigoureusement penser son rapport à la pratique sous forme d'application, comme c'est le cas pour les sciences de la nature. Cela tient sans doute profondément au fait que si un savoir linguistique spéculatif peut évidemment être de nature empirique, et, en ce qui concerne les langues naturelles, il l'est généralement ${ }^{4}$, il a difficilement pu, jusqu'à l'apparition récente de l'informatique, être de nature expérimentale.

Pour l'histoire des idées linguistiques, le seuil de l'écriture est fondamental. Quelle que soit la civilisation, nous rencontrons toujours les éléments d'un passage de l'épilinguistique au métalinguistique, qu'il s'agisse de l'apparition des mots métalinguistiques (dire, etc.), de certaines pratiques langagières, de spéculations sur l'origine du langage, ou sur l'identité et la différenciation linguistiques. Mais, à notre connaissance, nous ne rencontrons dans aucune civilisation orale un corps de doctrine élaboré en relation avec les arts du langage, même là où nous pouvons remarquer que certains individus sont spécialisés dans le rôle de traducteurs ou de "poètes". Nous pouvons certainement rencontrer dans des civilisations traditionnelles sans écriture un corps de doctrine, comme c'est le cas chez les Bambara (cf. Bertaux*, 1985, où l'on trouvera une analyse comparée des structures de la représentation métalinguistique) ou chez les Dogon, seul exemple, à notre connaissance, ayant bénéficié d'une analyse approfondie (Calame-Griaule, 1965). Chez ces derniers, la parole $(s j)$ est distinguée des cris ( $m i$, voix vivante) et des bruits (signe); elle divise le monde entre les êtres qui la possèdent et ceux qui ne la possèdent pas. Pour être émise, elle doit être composée. Elle prend son eau dans les clavicules, le sang, et finalement dans la salive; son air dans les poumons; son feu dans le cour; la terre, signification du discours, vient de tout le corps, mais plus particulièrement du cerveau. L'ébullition de l'eau dans le foje lui communique un mouvement vibratoire de façon à ce qu'elle puisse atteindre, en suivant un chemin qui dépend de sa qualité, l'auditeur. Celui-ci l'assimile au travers du tympan (sugúru tónu, dent de l'oreille); arrivée au larynx,

\footnotetext{
- Pour plus de précisions concernant les références placées entre parenthèses dans cet article, se reporter à la Bibliographie, p. 440-441.

4. Un savoir spéculatif (une représentation) est de nature empirique si sa valeur de vérité dépend d'une ou plusieurs assertions factuelles. En ce sens, parmi les sciences du langage, la logique n'est pas une discipline empirique. La grammaire générale et la grammaire comparée le sont, quoique de façon differente.
} 
elle se refroidit, se condense et reprend sa forme liquide (CalameGriaule, 1965, p. 58-74). Les paroles - les façons de dire - sont classées en fonction des circonstances de leur apparition mythique et selon un système de correspondances symboliques qui leur associe une technique, une institution, une plante, un animal ou une partie du corps humain (ibid., p. 104). Elles sont représentées graphiquement, mais loin d'être une écriture pictographique qui pourrait avoir tendance à se styliser, le dessin au contraire se complique et se surcharge, vivant en quelque sorte de sa vie propre. Si le dogon parle du langage de façon complexe et codifiée (le classement est le fondement d'une pragmatique contraignante), ce n'est pas qu'il dégage l'ordre du symbolique de l'ordre du réel, c'est bien plutôt que le réel est tout entier symbolique. Les paroles que l'homme échange avec sa femme avant l'acte sexuel sont diversement composées selon qu'elles sont "bonnes " ou "mauvaises"; ce sont elles qui, jointes au sperme, produiront le fœtus ou le sang trop cuit des menstrues. Si la parole fait des choses, elle ne le doit pas à une quelconque performativité, mais à sa composition matérielle. Les paroles sont en fait des choses parmi les choses. C'est ce qui, à nos yeux, explique une situation apparemment paradoxale. Calame-Griaule a décrit avec soin l'art du langage chez les Dogon (op. cit., p. 447-501) qui disposent d'une littérature orale assez riche. S'ils ont conscience d'une différence entre la parole ordinaire et la parole poétique, ils n'ont pas de mots pour nommer la seconde. Leur savoir métalinguistique leur permet tout juste de dire qu'elle a "plus d'huile" que la première, pas d'expliquer comment faire une strophe ou un refrain : ce savoir reste du domaine épilinguistique. Il en va de même pour la pédagogie des langues, dévolue par alternance à la femme et à l'homme lorsqu'il s'agit de la langue maternelle. La situation ethno-géographique incite à apprendre des langues étrangères, dont la connaissance est très prisée (op. cit., p. 260-261). Cette situation se reflète dans un mythe de l'origine immédiatement plurilingue. Binou Sérou, le premier homme à avoir reçu la révélation de la parole, a reçu toutes les langues, au nombre symbolique de douze (ibid., p. 98-99). Mais là encore le savoir métalinguistique mythique ne se connecte pas à la pratique et le savoir-faire épilinguistique ne se transforme pas en une technique verbalisée. Or, c'est cette transformation qui marque la naissance d'un véritable savoir linguistique, lorsque le métalangage prend en charge les manipulations effectuables sur le langage en lui-même. Tout semble montrer qu'il n'existe pas de véritable savoir grammatical oral, comme en témoigne au reste l'étymologie du mot. La linguistique populaire, dans son état de pensée sauvage, appartient à un autre registre.

Le processus d'apparition de l'écriture est un processus d'objectivation 
du langage, c'est-à-dire de représentation métalinguistique, considérable et sans équivalent antérieur. Il nécessite l'émergence de techniques autonomes et entièrement artificielles; il produit l'apparition d'un des tout premiers métiers du langage dans l'histoire de l'humanité, et vraisemblablement (nous manquons d'informations) de traditions pédagogiques. Mais si l'écriture joue un rôle fondamental dans l'origine des traditions linguistiques, ce n'est pas en elle-même, c'est au cours d'un processus historique complexe. Quand nous parlons d'origine, il ne s'agit évidemment pas d'un événement, mais d'un processus que nous pouvons enfermer dans un intervalle temporel ouvert, parfois considérablement long. L'origine d'une tradition peut être spontanée ou résulter d'un transfert technologique. La tradition latine est le résultat d'un transfert, de même la grammaire hébraïque, ainsi que l'étude des vernaculaires européens, amérindiens, africains, etc. Ce transfert peut accompagner un transfert des techniques d'écriture, comme cela s'est passé au Japon, après l'importation des caractères chinois au $v^{e}$ siècle de notre ère. Il peut s'effectuer sur le substrat d'une tradition spontanée (cas de l'hébreu). Il peut être plus diffus, comme il est possible que cela soit le cas pour l'arabe, avant la diffusion massive des philosophes grecs au $x^{e}$ siècle; nous parlerons dans ce cas d'influence. Nous disposons de peu de cas d'apparition spontanée, c'est-à-dire indépendante, de tradition linguistique : deux sont demeurées embryonnaires, les traditions babyloniennes et égyptiennes, trois ont abouti dans le long terme, les traditions indienne, chinoise et grecque, la dernière étant la source de toute la tradition occidentale. Au début du $\mathrm{xx}^{\mathfrak{e}}$ siècle, on peut considérer qu'un processus de domination et de transfert de la tradition occidentale vers toutes les autres traditions est achevé, d'où résulte une homogénéisation relative des grands traits spéculatifs du savoir linguistique. La tradition indienne n'a été connue de l'Occident qu'au XIX ${ }^{e}$ siècle; et il n'y a jamais eu d'emprunt durable de cette dernière tradition à la tradition chinoise, contrairement à ce qui s'est passé pour d'autres technologies (par exemple, la boussole). Cela tient à ce que, dans le cas du langage, le transfert technologique suppose une bonne connaissance de la langue source et de sa culture, laquelle n'a eu lieu que tardivement dans le cas du chinois, mais aussi à la spécificité d'une tradition, inéluctablement marquée par le rapport à une écriture radicalement étrangère aux modes d'expression occidentaux.

Soit donc l'écriture. Comment nait spontanément à partir de là une tradition de savoir linguistique? Chez les Babyloniens, les Égyptiens ou les Chinois, un processus analogue semble s'être réalisé. L'écriture produit des textes, en particulier des textes littéraires. Même s'il est évident que toute écriture suppose des normes, notamment stylistiques, 
elle ne semble pas produire spontanément une réflexion sur la nature du langage, voire un savoir codifié sur les procédés langagiers, à partir de ses techniques propres. Ce qui apparaît en premier, ce sont des listes de mots (ou de caractères pour le chinois). Leur utilité n'est pas très claire au départ; elles ont peut-être un rôle mnémotechnique. Mais ce qui fait véritablement démarrer la réflexion linguistique, c'est l'altérité, envisagée essentiellement du point de vue de l'écrit. Ainsi les Égyptiens listent-ils d'abord des mots non autochtones; les scribes utilisent aussi différentes conventions pour noter l'usure phonétique. Au III $^{\mathrm{e}}$ siècle avant notre ère, les Chinois étudient leurs caractères pour pouvoir lire les textes anciens ; les considérations phonétiques apparaissent, lorsqu'au $\mathrm{I}^{\text {er }}$ siècle de notre ère le développement du bouddhisme amène à transcrire des textes sanskrits. Chez les Babyloniens également nous trouvons des listes de mots, trois millénaires avant notre ère. Autrement dit, l'essor du savoir linguistique a sa source dans le fait que l'écriture fixant le langage objective l'altérité, que celle-ci provienne de l'ancienneté, de la nécessité de transcrire une autre langue, voire de celle de déchiffrer un texte qu'on ne connait pas auparavant, comme cela semble être le cas en Grèce. Ce sont, en quelque sorte, la philologie et la lexicologie qui apparaissent d'abord. Jamais nous n'observons une tradition linguistique naître sur la base d'une maîtrise des langues. Il ne semble pas que la nécessité de communiquer pour les échanges commerciaux et politiques, qui entraîne nécessairement l'existence d'individus polyglottes (voire leur spécialisation sous forme d'interprètes), entraîne sur cette base, ni une spéculation qui dépasse les généralités sur la diversité linguistique, ni la transmission de techniques codifiées (manuels de traduction). Le bilinguisme ne semble jouer de rôle important que dans les cas de transfert culturel massif (cas grec/latin, chinois/japonais, latin/vernaculaires européens), ou dans celui de la permanence des langues mortes. L'immense empire hittite, polyglotte, qui a dû employer quantité de scribes à traduire les textes officiels, qui disposait de vastes bibliothèques et de l'héritage babylonien, n'a laissé que des listes de mots bi- ou trilingues (Hovdhaugen, 1982, p. 17). Ceci est à mettre en rapport avec le fait que les Chinois ne se sont guère intéressés qu'au chinois et que les deux traditions dont les résultats ont été les plus achevés, tant du point de vue de la diversité que de la complexité, l'indienne et la grecque, se sont construites sur des bases monolingues. La première apparition connue de paradigmes systématiques et d'une terminologie grammaticale ne contredit pas ce schéma. Elle a lieu au début du second millénaire dans des textes bilingues sumérien/akkadien : à cette époque, le sumérien est pratiquement une langue morte. Autant dire que la première analyse grammaticale n'est 
pas née de la nécessité de parler une langue quelconque, mais de celle de comprendre un texte. De nos jours, la grammaire est enseignée à l'école aux enfants qui maitrisent encore mal leur langue ou qui ont à apprendre une langue étrangère. Cela tient autant au développement du système scolaire lui-même qu'à celui de la grammaire. Dans les temps les plus anciens, on n'a jamais eu spontanément l'idée de faire une grammaire - un corps de règles expliquant comment construire les mots, fût-ce sous la forme implicite de paradigmes - pour apprendre à parler. La tradition arabe, comme la tradition indienne, comporte bien le topos d'une invention de la grammaire, pour guider la langue parlée et corriger les fautes. Il ne s'agit probablement que d'un mythe fondateur, constitué après coup, et en tout cas après la constitution d'une tradition écrite : si les grammairiens sanskrits et arabes se préoccupent de la prononciation, il s'agit d'abord de la prononciation d'un texte écrit. Spontanément on apprend à parler en parlant. Mais il y a une chose qui est sûre, c'est que dès qu'existe un système d'écriture, il faut l'apprendre. Contrairement à la compétence linguistique, c'est un système déjà tout formé qui est transmis. C'est sans doute ce qui explique le rôle de l'écriture dans le développement des savoirs linguistiques. La grammatike grecque, qui naît au tournant des $v$ et IV siècles avant notre ère, n'est qu'un apprentissage élémentaire de la lecture et de l'écriture.

Ce que nous appelons une grammaire, et qui a été, pendant deux millénaires, l'une des formes de savoir linguistique les plus travaillées en Occident, repose sur le découpage de la chaîne parlée (ou écrite, dans la plupart des cas), c'est-à-dire la reconnaissance des unités et - contrairement à la lexicographie - leur projection sur une dimension paradigmatique qui rompt avec la linéarité de cette chaîne. On a vu comment un savoir de type grammatical pouvait naître de la pratique textuelle sur la base d'une pratique de l'écriture. Si ces éléments jouent toujours un rôle, ils ne sont pas nécessairement décisifs. La reconnaissance des unités tient souvent à d'autres pratiques et à d'autres savoirs. Le cas est particulièrement clair pour les noms personnels (cf. Houis, 1963), dont le savoir, l'imposition et la manipulation dépendent des formes de socialisation et des structures de parenté, comme Lévi-Strauss l'a montré dans La Pensée sauvage (1962), notamment. Chez les Wik Munkan d'Australie, cela donne lieu à une riche nomenclature : on distingue les vrais noms propres (nämp), les termes de parenté (nämp kämpan), les sobriquets (nämp yann), et les trois noms personnels de l'individu, le nom "ombilical " (nämp kort'n), le grand nom (nämp pi'in) et le petit nom (nämp mäny) (cf. Lévi-Strauss, op. cit., Paris, Plon, 1985 , p. 221). Le système est trop limité, trop particulier (il est lié 
à la motivation des signes), pour conduire à un savoir linguistique général, même si on peut montrer que le système des noms propres (ceux des dieux en particulier) est à la source des spéculations linguistiques grecques, notamment d'ordre étymologique (cf. Gambarara, 1984a, 1984b). En fait, il semble que la reconnaissance des unités, et la formulation de règles à leur propos, puisse naître spontanément sur la base d'une maîtrise de l'énonciation, laquelle dépend évidemment du statut social de certains types de discours (débats politiques, rôle de la poésie, discussion juridique, etc.). C'est ce qui s'est passé en Grèce, à partir $d u v^{e}$ siècle avant notre ère. La maitrise de certains types de discours (poétique, rhétorique), leur adéquation à certains buts pragmatiques (convaincre, dire le vrai, c'est-à-dire rhétorique et logique), la spéculation sur les rapports du logos à l'Être (philosophie), se sont conjoints pour produire une théorie des parties du discours. La grammaire, à proprement parler, ne naît que plus tard à partir de ces éléments, deux siècles avant notre ère, dans l'atmosphère philologique de l'école d'Alexandrie. Tard venue, elle n'a évidemment pas pour but d'apprendre à parler. Selon Sextus Empiricus, Denys le Thrace définissait la grammaire comme "la connaissance empirique poussée le plus loin possible de ce qu'on lit chez les poètes et les prosateurs " (Contre les grammairiens, § 57). Toute décomposition de l'énoncé n'est pas ipso facto grammaticale; pour qu'elle le soit, il faut qu'elle rejoigne la morphologie. La tradition chinoise n'a pas connu de naissance spontanée (autochtone) de la grammaire. Elle a connu pourtant une réflexion sur les types d'unité, en fonction de leur signification et de l'adéquation de celle-ci à certains buts. Il y eut ainsi des spéculations sur le rapport du langage au réel. L'École des Noms (Ming Chia) distinguait le nom (ming) et l'actualité (shi), pour repérer (voire utiliser) des argumentations sophistiques dès le $v^{e}$ siècle avant notre ère ${ }^{5}$. Dans les "canons mohistes " qui forment la partie logique du Mo-tzu ( $v^{e}$ siècle avant notre ère), l'actualité est conçue comme ce dont on parle et le nom ce qui sert à en parler, ce qui peut être comparé à la distinction entre sujet et prédicat. Les noms sont répartis en trois classes : les généraux, qui conviennent à toutes choses, les classificateurs (noms communs) et les noms propres ${ }^{6}$. Il n'y a rien là qui dépasse les buts de la recherche pragmatique de l'adéquation au réel. Pareillement la distinction, née dans la poétique, entre mots pleins et mots vides, n'aboutit pas à une théorie des parties du discours. Cela tient à ce que le chinois n'a pas de morphologie. Le cas du japonais, langue qui connaît suffixes et

5. Cf. Fung YU-LAN, A Short History of Chinese Philosophy (1948), paperback edition, New York, The Free Press, 1966, p. 80-83.

6. Ibid., p. 119. 
conjugaisons, le montre a contrario : bien que la tradition linguistique résulte d'un transfert à partir du chinois, elle a élaboré spontanément un système des parties du discours, comme l'a noté le missionnaire Joao Rodriguez au XVII siècle (Maës, 1982, p. 19).

Les causes agissant sur le développement des savoirs linguistiques sont extrêmement complexes. On peut noter pêle-mêle : l'administration des grands États, la littérarisation des idiomes et son rapport à l'identité nationale, l'expansion coloniale, le prosélytisme religieux, les voyages, le commerce, les contacts entre langues, ou le développement de connaissances connexes comme la médecine, l'anatomie ou la psychologie. Le purisme et l'exaltation identitaire, avec leur accompagnement de constitution/préservation d'un corpus littéraire (qu'il soit religieux ou profane), sont, par exemple, des phénomènes quasi universels dans la constitution, spontanée ou par transfert, des savoirs linguistiques. Leurs causes peuvent cependant être très diverses : appareil d'État et administration, expansion d'une religion, émergence d'une conscience nationale avec ou sans unification politique, dispersion d'un peuple, etc. Avant le $\mathrm{XIX}^{e}$ siècle européen et le développement de la phonétique expérimentale, on ne compte pas d'innovation technologique qui ait agi sur la connaissance du langage. Il faut mettre à part, bien entendu, les techniques d'écriture et les innovations portant sur le support ou la diffusion de l'écrit. De ce point de vue, que dans les vieux alphabets sémitiques (surtout l'araméen, mais aussi le phénicien) l'emploi du pinceau et du papyrus ait modifié profondément le tracé des lettres et leurs agencements (Février, 1959, p. 73) n'est qu'anecdotique. Mais l'apparition de l'imprimerie, dans le contexte de la diversité des langues dans les nations européennes et du développement du capitalisme marchand (les caractères mobiles d'imprimerie apparaissent en Chine ${ }^{7}$ au $\mathrm{XI}^{\mathrm{e}}$ siècle) est un moteur décisif pour la grammaticalisation et la standardisation des vernaculaires européens. Les grandes transformations des savoirs linguistiques sont avant tout des phénomènes culturels qui affectent le mode d'existence d'une culture autant qu'ils en proviennent.

Avec toute la prudence qui s'impose, on pourra certes retracer des analogies dans le développement des grandes traditions linguistiques indépendantes. Mais il faudra aussi remarquer des éléments idiosyncrasiques, qui ont en quelque sorte le caractère général d'événements uniques susceptibles d'infléchir le développement ultérieur. La phonétique de Panini est un de ces éléments/événements. Ce qui se passe en Europe

7. Cf. Joseph Needham, La Tradition scientifique chinoise, Paris, Hermann, 1974, p. 284. 
à la Renaissance en est un autre, quoiqu'il vaudrait mieux parler dans ce cas d'un macro-événement à structure complexe. D'abord, lorsque les vernaculaires européens sont systématiquement grammaticalisés à cette époque, ils le sont sur la base d'une orientation pratique qui s'est définie très lentement à partir des Artes de la tradition gréco-latine : une grammaire peut avoir pour but l'apprentissage des langues étrangères. Dans ce contexte, les contacts linguistiques deviennent l'un des éléments déterminants des savoirs linguistiques codifiés et les grammaires deviennent la pièce maîtresse d'une technologie langagière. Ensuite, le développement du livre imprimé donne à ce phënomène une diffusion incomparable. Enfin, l'exploration de la planète, la colonisation et l'exploitation de vastes territoires entament le long processus de description, sur la base de la technologie grammaticale occidentale, de la plupart des langues du monde. Cette entreprise ramifiée de savoir multilingue - dans le contexte de laquelle naîtront aussi bien la grammaire générale que la grammaire comparée - est aussi unique dans l'histoire de l'humanité que la physique mathématique galiléo-cartésienne, à laquelle elle est sans conteste homogène ${ }^{8}$, ne serait-ce que par l'idée de déterminer des régularités qui seraient, non pas des prescriptions de l'usage culturel des langues, mais des nécessités inhérentes à leur nature ou des «lois» de leur développement historique. Quelle qu'ait été l'importance des intérêts culturels, politiques et économiques en jeu, aussi bien lors de sa naissance qu'au cours de son développement, sa caractéristique essentielle est d'être déterminée, assez vite, essentiellement par un intérêt de connaissance. Dans aucune autre culture n'est apparu spontanément ce projet de décrire les langues du monde, qu'on voit se réaliser avec des ouvrages comme le Mithridates (1555) de Konrad Gesner. Dans aucune autre culture la maitrise des langues n'a abouti à ce savoir désintéressé et abstrait qu'est la grammaire comparée,

8. Dans une problèmatique bachelardienne, on se préoccupe de savoir quand a eu lieu la rupture qui fait passer le savoir linguistique au statut de science, conçue selon le modèle de la physique galiléco-cartésienne. La grammaire générale - qui lui est contemporaine - s'est réclamée de ce modèle; les comparatistes - dont le modèle est plutòt la biologie - ont proclamé pour leur propre compte le statut scientifique à l'exclusion de tout ce qui les a précédés. La tradition historiographique tient - la plupart du temps - pour accordée la vérité de cette proclamation. Le problème est de la justifier épistèmologiquement. Milner (1978, p. 31-32) le fait à partir de la naissance d'un système de notation symbolique, qui existerait pour la grammaire comparée et pas pour la grammaire générale. On peut toutefois faire remarquer que i) on a des exemples de notation symbolique qui apparaissent sporadiquement, par exemple, dans la grammaire arabe ou la grammaire générale, ii) l'absence d'une notation symbolique ne préjuge en rien du caractère formel d'une discipline, puisque la logique médiévale n'en a jamais employée, se contentant de formuler les règles à l'aide d'un métalangage (on retrouve la mème procédure dans la logique de Port-Royal). 
poursuivie durant tout le $\mathrm{XIX}^{e}$ siècle, par des professionnels dans le lieu clos des universités.

Une telle situation - qui est l'aboutissement d'un processus qui a ses racines dans la naissance même de la tradition occidentale - suppose des ruptures et des délimitations de domaine. La première est assez simple, elle concerne la séparation de la technique et de la théorie. Elle est acquise dès l'existence de la grammaire, dont l'a-théoricité est au départ assez claire face à ce type spécifiquement occidental de spéculation abstraite qu'est la philosophie. Tout se brouille dès le Moyen Âge, quand la grammaire elle-même se déplace vers le domaine spéculatif. Le savoir linguistique abstrait - celui qui se réfléchit luimême comme ce qu'on appelle une science - va avoir à se définir dans une relation de délimitation/opposition par rapport à la logique et à la philosophie. Pour la première, qui s'occupe de la façon dont on peut passer d'un énoncé vrai à d'autres énoncés vrais, la question paraît rapidement tranchée. Pourtant, parce que la logique aboutit nécessairement à des représentations de la forme des énoncés des langues naturelles, le transfert et le bricolage de concepts entre elle et la grammaire a toujours marqué les grandes étapes de leurs évolutions simultanées. Au point qu'au $\mathrm{XIX}^{\mathrm{e}}$ siècle, lorsque l'exigence d'une autonomie du savoir linguistique s'est faite jour, pour des raisons autant institutionnelles que théoriques, les linguistes ont inventé le péché de "logicisme", faute qui consiste à importer de la logique dans la « linguistique " (sur ce concept, cf. Coseriu, 1967). Pour la philosophie - dont la logique fait traditionnellement partie - c'est plus compliqué. Si le langage est matière à philosopher (cf. Hacking, 1975), c'est pour des raisons d'essence. La philosophie occupe le terrain des spéculations mythiques : pourquoi y a-t-il du langage plutôt que rien (Milner, 1978, p. 36)? Le domaine traditionnel de la philosophie est celui des rapports du langage à la pensée, au vrai et au réel, quelles que soient les raisons qui font que tel ou tel philosophe (par exemple Kant) ait pu envisager d'économiser tout détour par le langage. La séparation d'avec la philosophie n'a d'autre recours que le déni de philosophie, le refus des questions d'essence, d'origine et d'universalité. Elle a lieu par exclusion dans la non-science et le fantastique (Auroux et al., éds, 1985), exclusion qui n'est que l'envers du processus par quoi, dans l'Europe $\mathrm{du} \mathrm{XIX}^{e}$ siècle, une linguistique institutionnellement autonome peut se donner la positivité d'un objet spécifique : les langues en elles-mêmes et pour elles-mêmes.

Sylvain Auroux, C.N.R.S. 


\section{BIBLIOGRAPHIE}

Aarsleff (Hans), Kelly (Louis G.), Niederehe (Hans-Josef), éds, 1987, Papers in the History of Linguistics, Amsterdam, John Benjamins.

Amirova (I. A.), Ol'Chovikov (B.A.), Rozdestvenskis (Ju. V), 1980, Abriß der Geschichte der Linguistik, VEB Bibliographisches Institut Leipzig (original russe, Moscou, Nauka, 1975).

ANDRESEN (Julie), 1978, "François Thurot and the First History of Grammar ", Historiographia Linguistica, V, 1-2, p. 45-57.

ARENS (Hans), 1957, Sprachwissenschaft: Der Gang ihrer Geschichte von der Antike bis zur Gegenwart, Friburg/Munich, K. Alber.

Auroux (Sylvain), 1987a, «The First Uses of the French Word 'Linguistique ' (1812-1880) ", in AARSLEFF et al., éds, p. 447-459.

Auroux (Sylvain), 1987b, " Histoire des sciences et entropie des systèmes scientifiques ", in SCHMITTER, 1987, p. 20-42.

Auroux (Sylvain), Joly (André), Glatigny (Michel), Nicolas (Anne), Rosier (Irène), éds, 1984, Matériaux pour une histoire des théories linguistiques, Villeneuve-d'Ascq, Presses universitaires de Lille.

Auroux (Sylvain), Chevalier (Jean-Claude), JaCQues-Chaquin (Nicole), MarCHELlo-Nizia (Christiane), éds, 1985, La Linguistique fantastique, Paris, Clims/Denozl.

BARATIN (Marc), Desbordes (Françoise), 1981, L'Analyse linguistique dans l'Antiquité classique, Paris, Klincksieck.

BERTAUX (Christian), 1985, "Linguistique occidentale et divination Bambara ", in Auroux et al., éds, 1985, p. 367-377.

Calame-Griaule (Geneviève), 1963, « L'art de la parole dans les cultures africaines ", Présences africaines, 47, p. 73-91.

Calame-Griaule (Geneviève), 1966, Ethnologie et langage. La Parole chez les Dogon, Paris, Gallimard.

COSERIU (Eugenio), 1967, « Logicismo y antilogicismo en la gramatica ", in Teoria del Lenguaje y Linguistica General, Madrid, Gredos, p. 235-260.

CoserIU (Eugenio), 1969-1972, Die Geschichte der Sprachphilosophie von der Antike bis zur Gegenwart, 2 vols, Tubingen, Narr.

FEVrier (James G.), 1959, Histoire de l'écriture, $2^{e}$ éd., Paris, Payot.

GAMBARARA (Danièle), 1984a, « Réflexion religieuse et réflexion linguistique à l'origine de la philosophie du langage ", in Auroux et al., éds, 1984, p. $105-114$. 
Gambarara (Danièle), 1984b, Alla Fonti delle Filosofia del Linguaggio, Rome, Bulzoni.

GroTsCH (Klaus), 1982, Sprachwissenschaftsgeschischtsschreibung. Ein Beitrag zur Kritik und zur historischen und zur Selbstvergewisserung der Disziplin, Göppingen, Kümmerle Verlag.

Hacking (Ian), 1975, Why Does Language Matter to Philosophy?, Cambridge, C.U.P.

HARrIs (Roy), 1980, The Language Makers, Londres, Duckworth.

HouIs (Maurice), 1963, Le Nom individuel chez les Mossi, Dakar, I.F.A.N.

HovdHAUGEN (Even), 1982, Foundations of Western Linguistics, Oslo, Universitetsforlaget.

Hymes (Dell), ed., 1974, Studies in the History of Linguistics. Traditions and Paradigms, Bloomington/Londres, Indiana University Press.

Kelly (Louis G.), 1969, 25 Centuries of Language Teaching, Rowley, Newbury House Publishers.

KOERnER (Konrad), 1978, Western Histories of Linguistic Thought. An Annotated Chronological Bibliography, 1822-1976, Amsterdam, John Benjamins.

Koerner (Konrad), 1987, « Das Problem der Metasprache in der Sprachwissenschaftsgechichtsschreibung ", in SCHMITIER, éd., 1987, p. 63-80.

MAEs (Hubert), 1982, "Les parties du discours dans la tradition grammaticale japonaise avant le XIIr siècle ", Langages, 68, p. 17-26.

Milner (Jean-Claude), 1978, L'Amour de la langue, Paris, Le Seuil.

Mounin (Georges), 1967, Histoire de la linguistique des origines au xx siècle, Paris, P.U.F.

Oesterreicher (Wulf), 1977, " Paradigma und Paradigmwechsel - Thomas S. Kuhn und die Linguistik», Osnasbrücker Beiträge zur Sprachtheorie, 3, p. 241-284.

PARret (Hermann), éd., 1976, History of Linguistic Thought and Contemporary Linguistics, Berlin/New York, De Gruyter.

PEARson (Bruce), 1977, "Paradigms and Revolutions in Linguistics", Lacus Forum, 4, p. 384-390.

Percival (Keith), 1976, "The Applicability of Kuhn's Paradigms to the History of Linguistics ", Language, 52, p. 285-294.

RoBiNs (Robert H.), 1967, A Short History of Linguistics, Londres, Longmans (trad. franç. Paris, Le Seuil, 1976).

SCHMITER (Peter), 1982, Untersuchungen zur Historiographie der Linguistik, Tubingen, Gunter Narr.

SChmitter (Peter), éd., 1987, Geschichte der Sprachtheorie. 1 : Zur Theorie und Methode der Geschichtsschreibung der Linguistik, Tübingen, Gunter Narr.

STross (B.), 1974, "Speaking of Speaking : Tenejapa Tzeltal Metalinguistics ", in R. BAUMAN, J. SHERzER, éds, Explorations in the Ethnography of Speaking, Cambridge, C.U.P., p. 213-239. 